\title{
ROS-mediated JNK/p38-MAPK activation regulates Bax translocation in Sorafenib-induced apoptosis of EBV-transformed B cells
}

\author{
GA BIN PARK $^{1 *}$, YUNOCK CHOI ${ }^{1 *}$, YEONG SEOK KIM $^{1}$, HYUN-KYUNG LEE $^{2}$, \\ DAEJIN KIM $^{1}$ and DAE YOUNG HUR ${ }^{1}$
}

\begin{abstract}
${ }^{1}$ Department of Anatomy and Research Center for Tumor Immunology, Inje University College of Medicine, Busan 614-735; ${ }^{2}$ Department of Internal Medicine, Inje University Busan Paik Hospital, Busan 614-735, Republic of Korea
\end{abstract}

Received October 22, 2013; Accepted December 2, 2013

DOI: $10.3892 / \mathrm{ijo} .2014 .2252$

\begin{abstract}
Sorafenib (SRF) is a multi-kinase inhibitor that has been shown to have antitumor activity against several types of cancers, but the effect of SRF on EBV-transformed B cells is unknown. We report that SRF can induce the apoptosis of EBV-transformed B cells through JNK/p38-MAPK activation. SRF triggered the generation of reactive oxygen species (ROS), translocation of Bax into the mitochondria, disruption of mitochondrial membrane potential, activation of caspase-9, caspase-3 and PARP, and subsequent apoptosis. Moreover, we found that SRF exposure activated the phosphorylation of JNK and p38-MAPK and suppressed the phosphorylation of PI3K-p85 and Akt. N-acetyl-l-cysteine (NAC) inhibited the activation of JNK and p38-MAPK. SP600125 and SB203580 blocked apoptosis and mitochondrial membrane disruption but did not affect ROS production after SRF treatment. These findings provide novel insights into the molecular mechanisms driving SRF-mediated cell death and suggest that SRF could be a potential therapeutic drug for the treatment of EBV-related malignant diseases.
\end{abstract}

\section{Introduction}

Epstein-Barr virus (EBV), also called human herpesvirus 4, is widespread in $>95 \%$ of the adult population and persists asymptomatically in all infected individuals for their entire

Correspondence to: Dr Dae Young Hur, Department of Anatomy and Tumor Immunology, Inje University College of Medicine, 633-165 Kaekum-2-dong, Jin-gu, Busan 614-735, Republic of Korea E-mail: dyhur@inje.ac.kr

*Contributed equally

Abbreviations: EBV, Epstein-Barr virus; SRF, sorafenib; ROS, reactive oxygen species; NAC, $\mathrm{N}$-acetyl-l-cysteine

Key words: EBV, B cell, sorafenib, ROS, JNK, p38-MAPK, Bax lives (1). Acute EBV infection is best known as the cause of infectious mononucleosis and its latent state is involved in several malignant disorders, including Burkitt's lymphoma, Hodgkin's lymphoma, non-Hodgkin's lymphoma, immunoblastic B lymphoma associated with HIV, T cell lymphoma, gastric carcinoma, nasopharyngeal carcinoma, rheumatoid arthritis, Sjogren's syndrome and multiple sclerosis (2)

Sorafenib (SRF; BAY43-9006, Nexavar) is a small molecule multi-kinase inhibitor that targets Raf kinases as well as diverse receptor tyrosine kinases including VEGFR1, VEGFR2, PDGFR, FLT-3 and c-Kit $(3,4)$. SRF was recently approved by the FDA for the treatment of hepatocellular carcinoma (5) and renal cell carcinoma (6) and is now undergoing phase II/III clinical trials $(7,8)$. SRF-based combination therapy has also been examined in several cancer clinical trials and studies $(9,10)$. Previous studies demonstrated that SRF has anti-proliferative, anti-angiogenic, and antitumor activity against various types of cancer and human xenograft models such as multiple myeloma, chronic myelogenous leukemia, chronic lymphocytic leukemia, acute myeloid leukemia, breast, renal, ovarian, colon, pancreatic, melanoma and non-small cell lung cancers (11). The apoptotic effects and molecular signaling mechanisms of SRF differed among these cancer cell lines. Generally, SRF inhibited the MEK/ERK pathway and decreased levels of the anti-apoptotic protein Mcl-1 (12), which is involved in resistance to anticancer drugs and is overexpressed in diverse leukemia and lymphoma. Furthermore, SRF-induced inhibition of the ERK pathway contributed to a decrease in Bcl- $\mathrm{X}_{\mathrm{L}}$ expression (13). SRF inhibited Hif-1 $\alpha /$ VEGF and downregulated the phosphorylation of mTOR/ ERK in hepatocellular carcinoma (14).

Mitogen-activated protein kinases (MAPKs) comprise ERK, JNK, and p38-MAPK, and mediate various signaling transduction pathways (15). The JNK/p38-MAPK pathway plays a central role in apoptosis, especially oxidative stressinduced apoptosis, whereas ERK is involved in cell proliferation, cell migration, cell differentiation and cell survival. These pathways play crucial roles in chemical-induced apoptosis. For instance, acanthoic acid leads to apoptosis of leukemia HL-60 cells by activating p38-MAPK without activating ERK or JNK (16). Curcumin provokes tumor cell death via 
activation of MAPKs (17). Berberine elicits apoptosis of HepG2 cells through p38-MAPK activation (18). However, it is unclear whether JNK/p38-MAPK signaling in involved in SRF-induced apoptosis.

Although SRF is an inhibitor of Raf/MEK/ERK, very little is known about whether or not SRF can induce apoptosis by activating alternative kinase pathways. It has been reported that SRF was able to elicit apoptotic cell death of human leukemia cells via a mechanism involving ER stress (19), but detailed molecular mechanisms were not elucidated. Therefore, we set out to investigate whether SRF can induce apoptosis through MEK/ERK-independent pathways in EBV-transformed B cells. We report the novel finding that SRF can induce apoptosis of EBV-transformed B cells by reactive oxygen species (ROS) generation, JNK/p38-MAPK activation, and Bax translocation.

\section{Materials and methods}

Preparation of EBV infectious culture supernatant and generation of EBV-transformed B cells. Cell-free EBV virions were prepared from culture supernatant of the B95-8 marmoset cell line. To establish EBV infection of B cells from normal peripheral blood mononuclear cells (PBMCs), PBMCs were isolated from peripheral blood of a healthy donor by Ficollpaque (Amersham Life Science, Buckinghamshire, UK) gradient centrifugation. PBMCs were added to EBV virion stock in a culture flask, and after a 2 -h incubation at $37^{\circ} \mathrm{C}$, RPMI-1640 culture medium (Hyclone) and $1 \mathrm{mg} / \mathrm{ml}$ of cyclosporine A (Sigma-Aldrich, St. Louis, MO, USA) were added to cells $\left(1 \times 10^{6}\right.$ cells $\left./ \mathrm{ml}\right)$. Cultures were incubated for 2-4 weeks. This study was approved by the Institutional Bioethics Review Board of the Medical College of Inje University and all donors provided informed consent.

Proliferation measurement by AlamarBlue. Cells $\left(5 \times 10^{4}\right.$ cells/well) were cultured in medium containing $10 \%$ FBS in 96-well plates. After $24 \mathrm{~h}$, cell proliferation was measured by AlamarBlue (Serotec Ltd., Kidlington, Oxford, UK) assay. AlamarBlue was added (10\% by volume) to each well and relative fluorescence was determined $9 \mathrm{~h}$ later using a SpectraMax M2e Multi-Detection Microplate reader (Molecular Devices, Sunnyvale, CA, USA; excitation, $530 \mathrm{~nm}$; emission, $590 \mathrm{~nm}$ ). Relative fluorescence unit (RFU) values are expressed as means \pm SEMs of three determinations.

Quantification of apoptotic cells by flow cytometry. The level of SRF (BAY43-9006, Nexavar; LC Laboratories, Woburn, MA, USA)-induced apoptosis in human EBV-transformed B cells (4 weeks, $5 \times 10^{5}$ cells $/ \mathrm{ml}$ ) and normal PBMCs was measured by flow cytometry using FITC-labeled Annexin-V (BD Biosciences, San Diego, CA, USA) and 7-AAD (BD Biosciences). In brief, to determine optimal conditions, experiments were performed using various concentrations of $\operatorname{SRF}(0,1,2.5,5$ and $10 \mu \mathrm{M})$ and various incubation periods $(2,4,8,16$ and $24 \mathrm{~h})$. To inhibit generation of ROS, cells were pretreated with NAC (N-acetyl-1-cysteine, $10 \mathrm{mM}$; SigmaAldrich) for $1 \mathrm{~h}$. To block activation of JNK or p38-MAPK, cells were pretreated with SP600125 (25 $\mu \mathrm{M}$; Calbiochem, San Diego, CA, USA) or SB203580 (10 $\mu \mathrm{M}$; Calbiochem) for $1 \mathrm{~h}$.
Cells were then harvested, washed in PBS, and incubated with Annexin-V and 7-AAD in Annexin-V binding buffer at room temperature for $15 \mathrm{~min}$ in the dark. Stained cells were analyzed using a FACSCalibur flow cytometer (BD Biosciences) running CellQuestpro software (BD Biosciences).

Detection of mitochondria membrane potential $\left(\Delta \Psi_{m}\right)$ and intracellular reactive oxygen species (ROS) generation. We detected changes in mitochondrial membrane potential $\left(\Delta \Psi_{\mathrm{m}}\right)$ using DiOC $_{6}$ (3,3'-dihexyloxacarboxyanine iodide; Molecular Probes, Eugene, OR, USA). Cells were treated with SRF or DMSO for $24 \mathrm{~h}$, harvested, washed twice in PBS, re-suspended in PBS supplemented with $\mathrm{DiOC}_{6}(20 \mathrm{nM})$, incubated at $37^{\circ} \mathrm{C}$ for $15 \mathrm{~min}$ in the dark, and then analyzed immediately by flow cytometry. Intracellular accumulation of ROS was examined by flow cytometry after staining with the fluorescent probe, DCFH-DA (2',7'-dichlorodihydro-fluorescein diacetate, $10 \mu \mathrm{M}$; Molecular Probes). DCFH-DA is deacetylated in cells by esterase to a non-fluorescent compound, DCFH, which remains trapped within the cell and is cleaved and oxidized by ROS in the presence of endogenous peroxidases to a highly fluorescent compound, DCF (2',7'-dichlorofluorescein). Briefly, EBV-transformed B cells were seeded in 6-well plates $\left(5 \times 10^{5}\right.$ cells $\left./ \mathrm{ml}\right)$ and pretreated with $10 \mu \mathrm{M}$ DCFH-DA for $30 \mathrm{~min}$ at $37^{\circ} \mathrm{C}$. Cells were then washed, re-suspended in RPMI-1640 media, and incubated with SRF or DMSO.

Western blot analysis. After treatment, cells were harvested and lysed in NP-40 buffer (Elpis Biotech, Daejeon, Korea) containing a protease inhibitor cocktail (AEBSF, aprotinin, bestatin hydrochloride, E-64, EDTA and leupeptin hemisulfate salt; Sigma-Aldrich). To address phosphorylation events, an additional set of phosphatase inhibitors (Cocktail II, sodium orthovanadate, sodium molybdate, sodium tartrate, and imidazole; Sigma-Aldrich) was added to NP-40 buffer (Elpis Biotech, Daejeon, Korea). Protein concentration was determined using a BCA assay kit (Pierce, Rockford, IL, USA). Proteins (10 $\mu \mathrm{g} / \mathrm{sample})$ were then heated for $5 \mathrm{~min}$ at $100^{\circ} \mathrm{C}$. Total cell lysates $\left(5 \times 10^{6}\right.$ cells/sample) were subjected to SDS-PAGE on $15 \%(\mathrm{w} / \mathrm{v})$ acrylamide gels under reducing conditions. Separated proteins were transferred to nitrocellulose membranes (Millipore Corp., Billerica, MA, USA), and membranes were blocked with 5\% skim milk followed by commercial western blot analysis. Chemiluminescence was detected using an ECL kit (Advansta Corp., Menlo Park, CA, USA) and the multiple Gel DOC system (Fujifilm). Primary antibodies against the following proteins were used: caspase-8, caspase-3, caspase-9, PARP, $\beta$-actin, Bcl-2, Bax, phospho-JNK (Thr ${ }^{183} / \mathrm{Tyr}^{185}$ ), JNK, phospho-p38-MAPK $\left(\mathrm{Thr}^{180} / \mathrm{Tyr}^{182}\right), \mathrm{p} 38-\mathrm{MAPK}$, phospho-ERK1/2 $\left(\mathrm{Thr}^{202} / \mathrm{Tyr}^{204}\right)$, ERK1/2, phospho-PI3K p85 (Tyr ${ }^{458}$ ), PI3K p85, phospho-Akt $\left(\mathrm{Ser}^{473}\right)$, Akt (Cell Signaling Technology, Beverly, MA, USA), COX-IV (Santa Cruz Biotechnology, Santa Cruz, CA, USA), and $\beta$-tubulin (BD Biosciences). Data were analyzed using Image J 1.38 software.

Measurement of Bax translocation. Following treatment, mitochondrial and cytosol cellular fractions were prepared using a Cytosol/Mitochondria Fractionation kit (Calbiochem). Approximately $1 \times 10^{7}$ treated or untreated cells were harvested 
by centrifugation at $600 \times \mathrm{g}$ for $5 \mathrm{~min}$ at $4^{\circ} \mathrm{C}$ and washed twice with cold PBS. Afterward, cells were re-suspended in $250 \mu \mathrm{l}$ cytosol extraction buffer containing protease inhibitor cocktail and $1 \mathrm{mM}$ dithiothreitol (DTT). After incubation on ice for $10 \mathrm{~min}$, cells were homogenized on ice using a Dounce tissue homogenizer. Homogenized cells were centrifuged at $700 \mathrm{x} \mathrm{g}$ for $10 \mathrm{~min}$ at $4^{\circ} \mathrm{C}$ and supernatants were collected. Supernatants were then centrifuged again at $10,000 \times \mathrm{g}$ for $30 \mathrm{~min}$ at $4^{\circ} \mathrm{C}$. The resulting supernatants were harvested and designated cytosolic fractions while pellets were re-suspended in $50 \mu \mathrm{l}$ mitochondria extraction buffer containing a protease inhibitor cocktail and $1 \mathrm{mM}$ DTT and designated mitochondrial fractions. All fractions were stored at $-80^{\circ} \mathrm{C}$ until use.

\section{Results}

SRF selectively induces apoptosis in EBV-transformed B cells but not in normal PBMCs. To investigate the effects of SRF on the proliferation of EBV-transformed B cells, cells were treated with various doses of $\operatorname{SRF}(1,2.5,5,10,20$ or $50 \mu \mathrm{M})$ for $24 \mathrm{~h}$ and then subjected to AlamarBlue assay. In the presence of SRF, EBV-transformed B cell proliferation decreased in a dose-dependent manner, suggesting that SRF has potential anticancer activity (Fig. 1A). SRF suppressed proliferation by $\sim 50 \%$ at a dose of $10 \mu \mathrm{M}$. We performed experiments to check whether this inhibitory effect of SRF on cell growth resulted from apoptotic cell death. As shown in Fig. 1B (upper panel), it is clear that treatment of cells with $\operatorname{SRF}(0,1,2.5,5,10,20$ or $50 \mu \mathrm{M})$ for $24 \mathrm{~h}$ increased the percentage of cell undergoing apoptosis (Annexin-V+/7-AAD ${ }^{+}$; 9.21, 13.95, 32.82, 43.04, and $65.76 \%$ respectively) compared with DMSO-treated cells (9.80\%), whereas up to $10 \mu \mathrm{M}$ SRF had no cytotoxic effects on normal human PBMCs (Fig. 1D). Fig. 1C (upper panel) shows cells that were treated with SRF for various time intervals; the percentages of Annexin-V and 7-AAD positive cells after incubation times of $2,4,8,16$ or $24 \mathrm{~h}$ were $6.75,6.84,6.07$, 21.44 and $39.90 \%$, respectively. Moreover, SRF disrupted $\Delta \Psi_{\mathrm{m}}$ significantly (Fig. 1B, lower panel), especially between 16 and 24 h (Fig. 1C, lower panel; from 46.93 to 64.18\%). Because the optimal dose and time of $\Delta \Psi_{\mathrm{m}}$ treatment were $10 \mu \mathrm{M}$ and $24 \mathrm{~h}$, we chose these conditions to examine protein alterations in SRF-induced apoptosis. Together, these results indicate that SRF preferentially targets cancerous EBV-transformed $\mathrm{B}$ cells.

SRF leads tocaspase-dependentapoptosis in EBV-transformed $B$ cells. To examine whether the strong apoptotic effect of SRF involved caspase activation, we analyzed cleavage of caspases and PARP by western blot analyses. SRF induced dose-dependent cleavage of caspase- $8,-9$, and -3 , followed by executioner capase- 3 activation and caspase-mediated cleavage of PARP, indicating that multiple caspases were activated in SRF-treated EBV-transformed B cells (Fig. 2A).

SRF-induced apoptosis involves alterations in the intracellular distribution of Bcl-2 and Bax translocation. Several genes have been reported to play an important role in modulating apoptosis. Abnormal expression of anti- and pro-apoptotic molecules after stimulation is one of the main mechanisms by which cell fate is determined in an apoptotic system. Accumulating evidence suggests that Bcl-2 family members play key roles in controlling apoptosis by acting as enhancers (e.g., Bax) or inhibitors (e.g., Bcl-2) of cell death (20-22). Accordingly, we monitored the expression of these molecules following SRF treatment using immunoblot analysis. We observed that SRF significantly downregulated protein levels of Bcl-2, whereas it had no effect on Bax expression (Fig. 2B). We separated mitochondrial and cytosolic fractions following SRF treatment to assess Bax translocation by western blot analysis. As depicted in Fig. 2C, there was a significant enhancement in translocation of Bax from the cytosol to mitochondria after SRF treatment compared with the control. Western blot analysis revealed that SRF caused an increase in the translocation of Bax to the mitochondria and an increase in the release of cytochrome $c$ to the cytoplasm, thus confirming the disintegration of $\Delta \Psi_{\mathrm{m}}$ after SRF exposure (Fig. 2C).

SRF induces sustained JNK and p38-MAPK activation and inhibits the PI3K/Akt survival pathway in EBV-transformed $B$ cells. MAPK signaling is associated with various cellular stresses and stimuli and has been shown to contribute to induction of apoptosis (23), whereas the PI3K/Akt pathway plays a critical role in the survival of various cancer cells, including EBV-transformed B cells (24). We thus examined the effects of SRF on MAPK and PI3K/Akt signaling and the role of these pathways in SRF-induced apoptosis of EBV-transformed B cells. Cells were exposed to SRF and the activity of ERK1/2, p38-MAPK, and JNK was assessed. Fig. 3A shows that SRF clearly induced activation of JNK and p38-MAPK after $3 \mathrm{~h}$ and phosphorylation was sustained for up to $12 \mathrm{~h}$ in a time-dependent manner, whereas the level of ERK1/2 phosphorylation did not change after SRF exposure. These results suggest that JNK and p38-MAPK are mediators of SRF-induced apoptosis. Moreover, we detected constitutive activation of the PI3K/Akt pathway in the control group, but SRF treatment decreased the phosphorylation of PI3K and Akt (Fig. 3B). To confirm the role of JNK and p38-MAPK in SRF-induced apoptosis, cells were exposed to SRF either alone or in combination with a specific JNK inhibitor (SP600125) and p38-MAPK inhibitor (SB203580) for $24 \mathrm{~h}$. As illustrated in Fig. 4A, both SP600125 and SB203580 pretreatment suppressed SRF-induced apoptosis (SRF, 73.56\%; with SP600125, 9.27\%; with SB203580, $11.22 \%$ ) and $\Delta \Psi_{\mathrm{m}}$ disruption (SRF, 76.49\%; with SP600125, $7.92 \%$; with SB203580, 8.32\%) effectively. These inhibitors completely abolished the activation of caspase-8, -9 and -3 , as well as degradation of PARP after SRF treatment (Fig. 4B) and blocked the translocation of Bax to mitochondria and the release of cytochrome $c$ to the cytosol (Fig. 4C). These data indicate that apoptosis caused by SRF treatment is dependent on the JNK/p38-MAPK pathway.

ROS is responsible for sustained activation of JNK and p38-MAPK and mitochondrial disruption by SRF. Reactive oxygen species, an early signal of apoptosis (25), are directly involved in the activation of caspases and MAPKs (26) and are responsible for the antitumor effects of several antitumor drugs. We evaluated whether SRF triggered intracellular ROS production and examined if ROS mediated JNK/p38-MAPK 
A

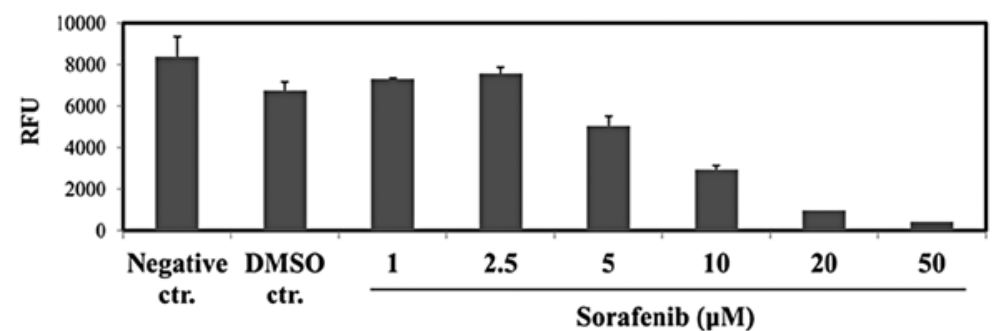

B

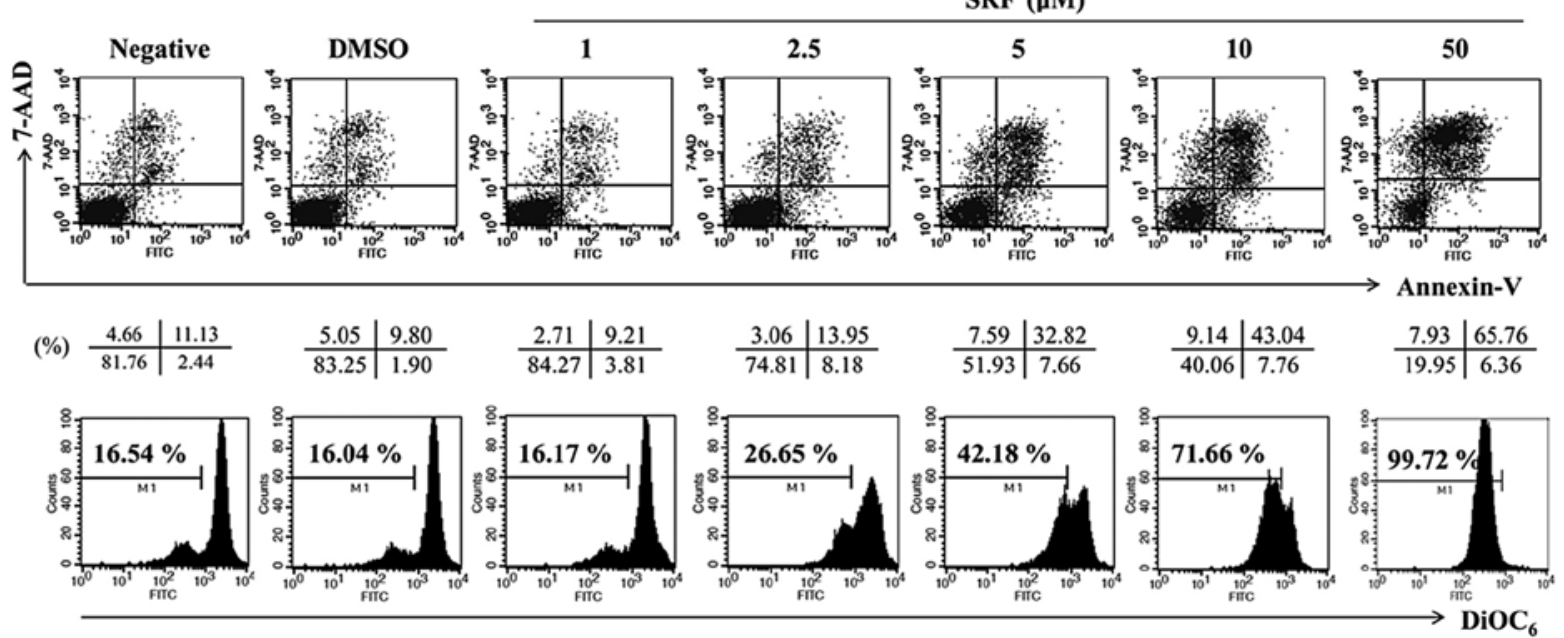

C

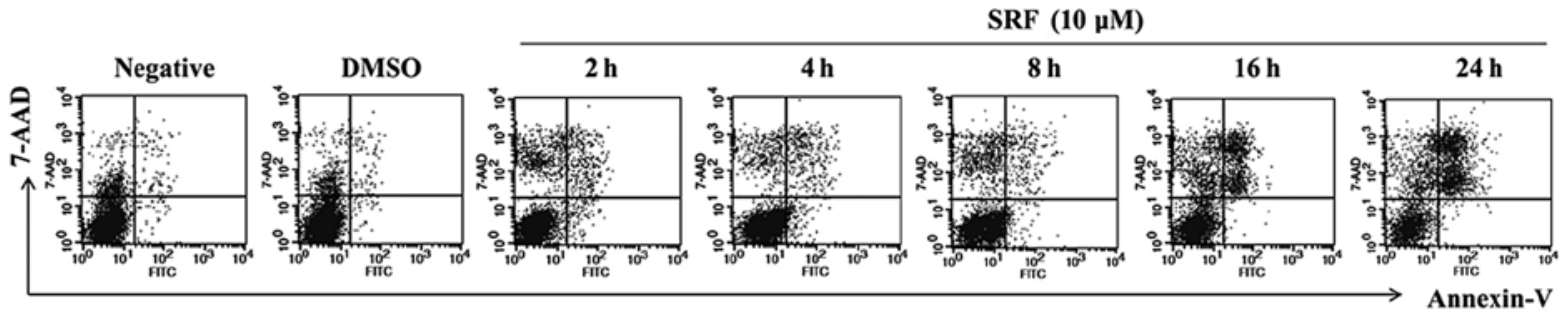

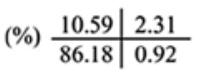

\begin{tabular}{l|l}
11.08 & 2.08 \\
\hline 86.04 & 0.80
\end{tabular}

\begin{tabular}{l|l}
15.71 & 6.75 \\
\hline 75.57 & 1.98
\end{tabular}

\begin{tabular}{c|c}
9.24 & 6.84 \\
\hline 80.24 & 3.69
\end{tabular}

\begin{tabular}{l|l}
16.07 & 6.07 \\
\hline 75.10 & 2.76
\end{tabular}

\begin{tabular}{l|l}
19.67 & 21.44 \\
\hline 58.08 & 0.81
\end{tabular}

\begin{tabular}{c|c}
18.20 & 39.90 \\
\hline 41.11 & 0.80
\end{tabular}
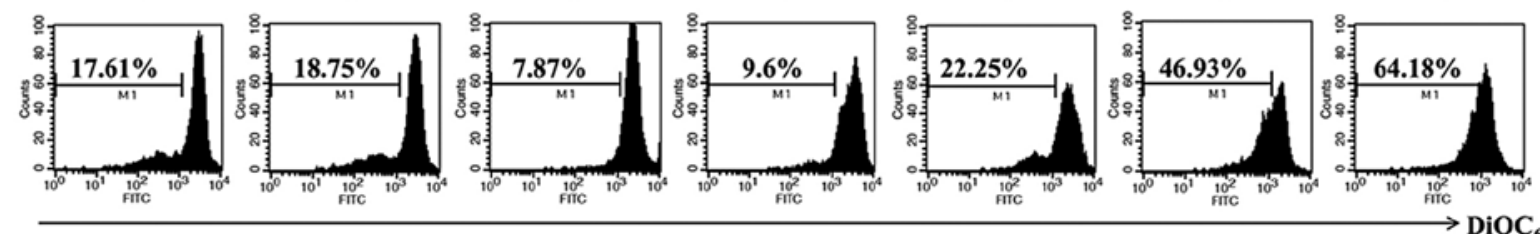

$\mathrm{DiOC}_{6}$

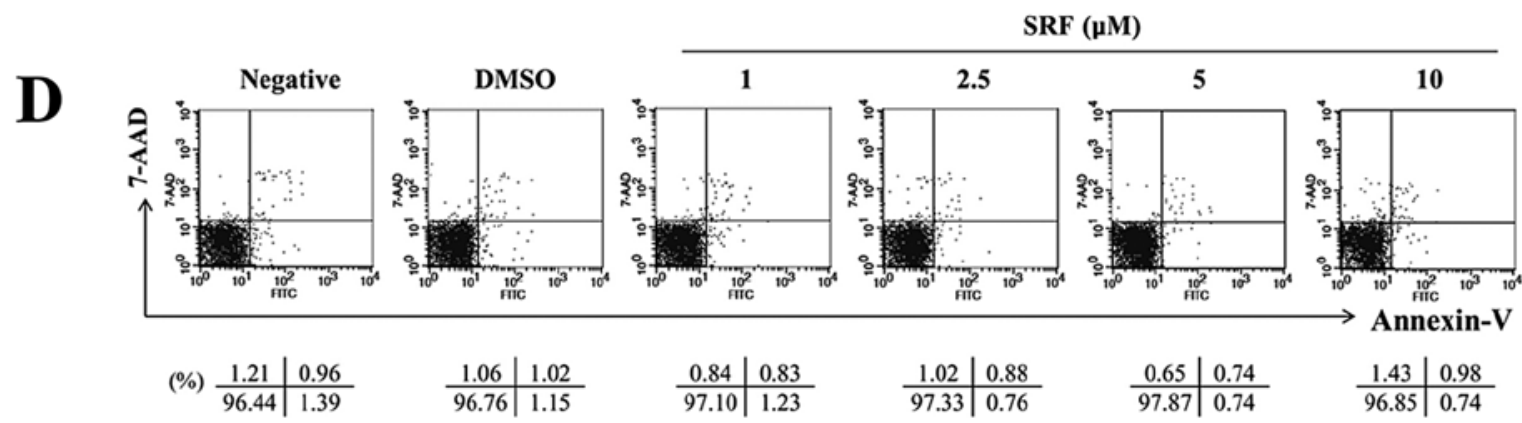

Figure 1. SRF induces apoptosis in a dose- and time-dependent manner in EBV-transformed B cells. (A) EBV-transformed B cells (5x10 ${ }^{4}$ cells/well) were cultured in 96-well plates and exposed to $\operatorname{SRF}(1,2.5,5,10,20$ and $50 \mu \mathrm{M})$ or DMSO. After $24 \mathrm{~h}$, cell proliferation was measured by AlamarBlue assay. RFU, relative fluorescence units. EBV-transformed B cells (B and C) and PBMCs (D) were treated with 1, 2.5, 5, 10 and $50 \mu \mathrm{M} \mathrm{SRF}$ for 2, 4, 8, 16 and $24 \mathrm{~h}$. The percentage of apoptotic cells was estimated by Annexin-V/7-AAD staining. Dot plot graphs showing the percentage of viable cells (Annexin-V/7-AAD), early-phase apoptotic cells (Annexin- $\left.\mathrm{V}^{+} / 7-\mathrm{AAD}^{-}\right)$, late-phase apoptotic cells (Annexin- $\mathrm{V}^{+} / 7-\mathrm{AAD}^{+}$), and necrotic cells $\left(\mathrm{Annexin}-\mathrm{V}^{-} / 7-\mathrm{AAD}^{+}\right)$. To measure disruption of $\Delta \Psi_{\mathrm{m}}$, cells were stained $\mathrm{DiOC}_{6}$. Diminished $\mathrm{DiOC}_{6}$ fluorescence indicates $\Delta \Psi_{\mathrm{m}}$ disruption. Percentages indicate the cell proportion in each bar. Results are representative of three independent experiments. 

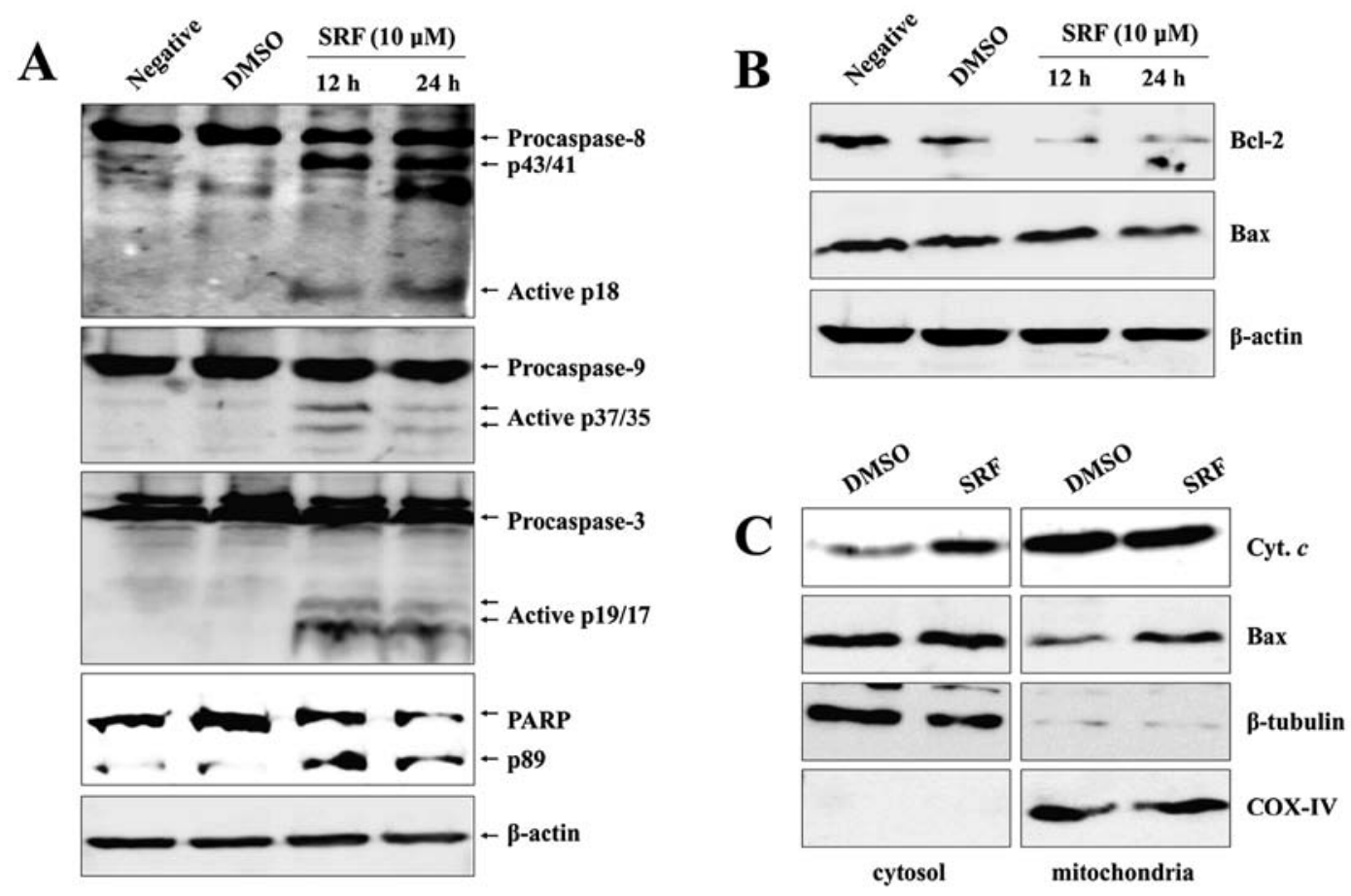

Figure 2. Effect of SRF on caspase activation and Bcl-2 family member expression. (A and B) EBV-transformed B cells were treated with $10 \mu \mathrm{M}$ SRF for the indicated times. Western blot analyses to determine expression of active casapse- 8 , -9 and -3 , PARP cleavage, Bcl-2 and Bax were performed to characterize the apoptotic response. $\beta$-actin was used to normalize the protein content. (C) Cells were harvested and then the amounts of cytochrome $c$ and Bax in the cytosolic and mitochondrial fractions were determined. The mitochondrial marker, COX-IV and the cytosolic marker, $\beta$-tubulin, were used to verify the purity of each fraction and were also used as loading controls. Cytosolic and mitochondrial fractionation was performed as described in Materials and methods. Results are representative of three independent experiments.

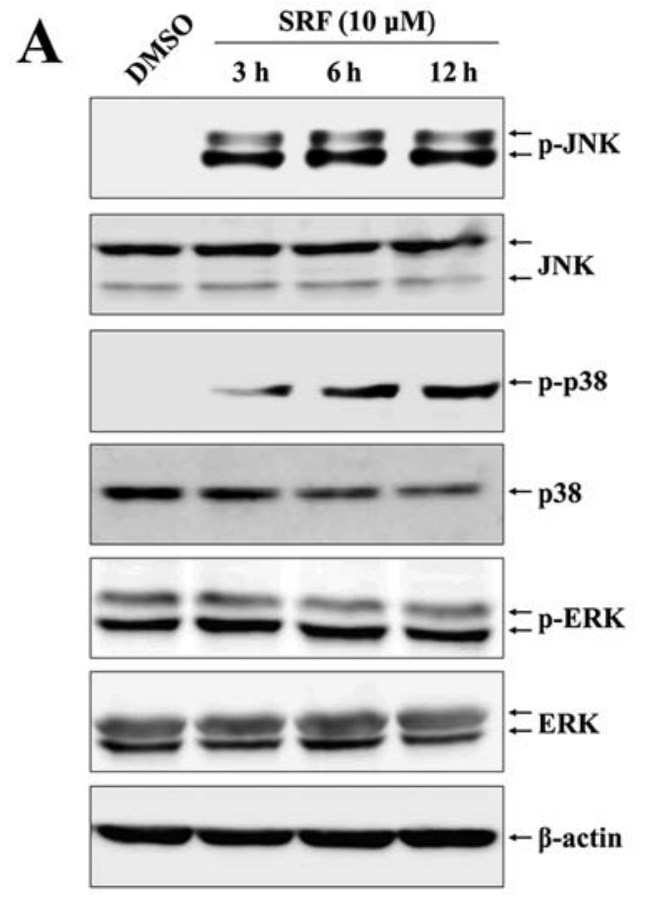

and PI3K/Akt signaling. Cells were exposed to $10 \mu \mathrm{M} \mathrm{SRF}$ for the indicated time periods, followed by loading with DCFH-DA to measure intracellular ROS levels. We found that SRF elicited a significant increment in DCF fluorescence within 1-2 h, and SRF-induced ROS levels were maintained

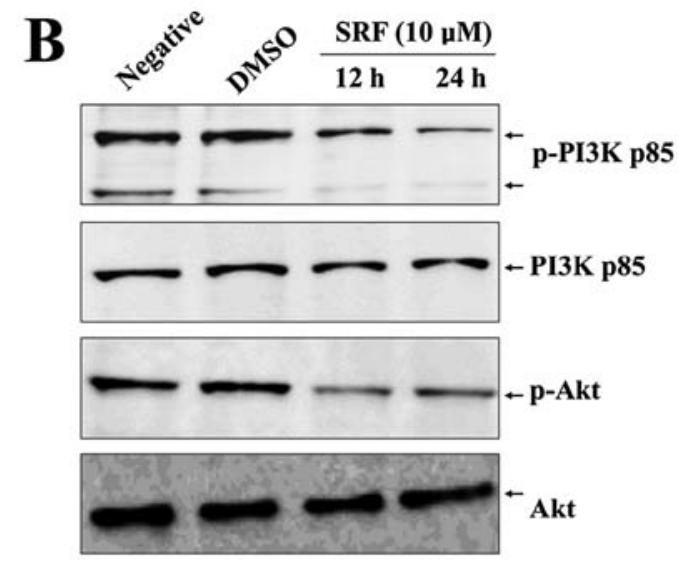

Figure 3. SRF causes rapid activation of JNK and p38-MAPK in EBVtransformed B cells. Cells were treated with $10 \mu \mathrm{M}$ SRF for the indicated time periods prior to cell lysis. Total protein was subjected to western blot analysis and successively immunoblotted for the following targets: (A) phospho-JNK, phospho-p38-MAPK and phospho-ERK1/2; (B) phospho-PI3K and phosphoAkt. JNK, p38-MAPK, ERK1/2, PI3K and Akt were used as internal controls. Results are representative of three independent experiments.

for up to $24 \mathrm{~h}$ (Fig. 5A). To verify the effect of SRF-induced ROS on the sustained phosphorylation of JNK/p38-MAPK and caspases during apoptosis, we pretreated cells with NAC, a scavenger of ROS, prior to SRF exposure. As shown in Fig. 5B, NAC significantly abrogated SRF-induced apoptosis 
A

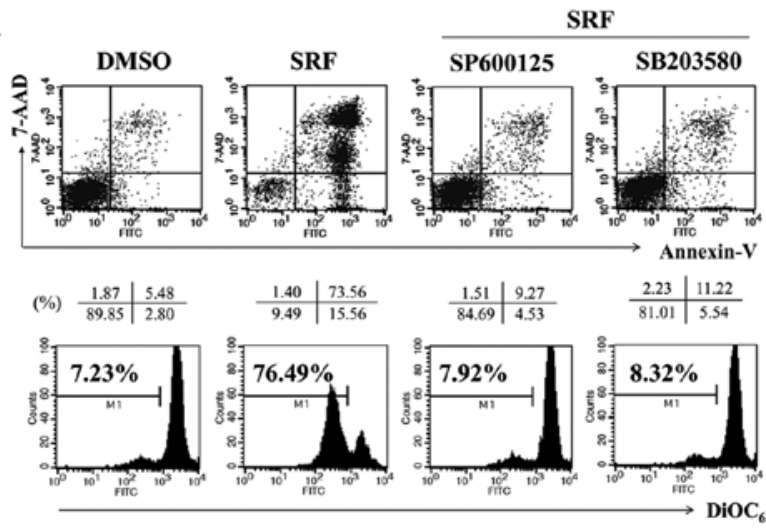

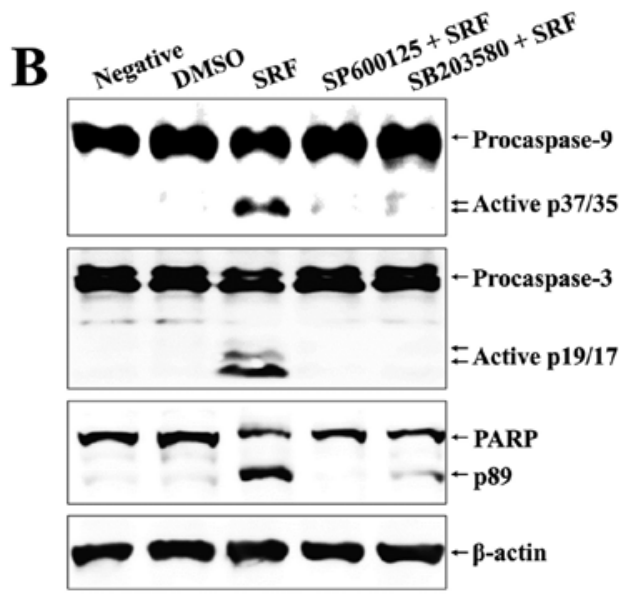

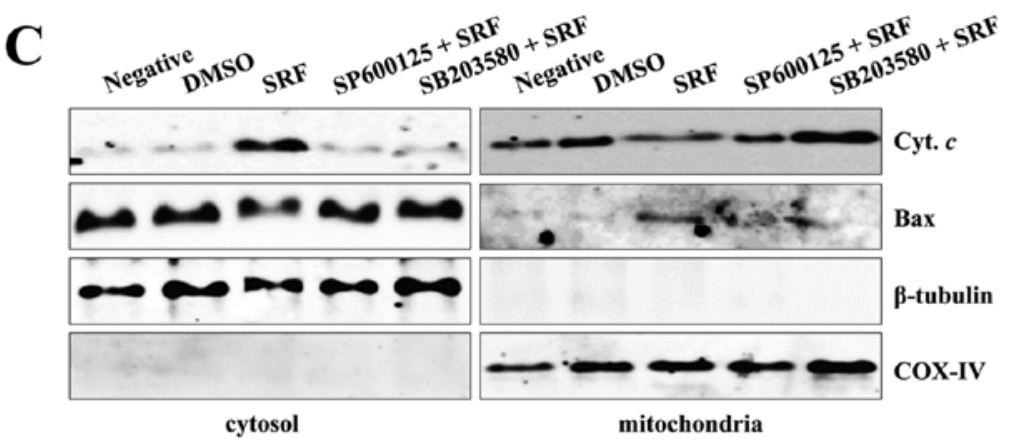

Figure 4. SRF enhances JNK and p38-MAPK activation in EBV-transformed B cells. Cells were pretreated with SP600125 (25 $\mu \mathrm{M})$ or SB203580 (10 $\mu \mathrm{M})$ for $1 \mathrm{~h}$. Cells were then washed with PBS and incubated with $10 \mu \mathrm{M} \mathrm{SRF}$ for $24 \mathrm{~h}$. (A) The number of apoptotic cells (Annexin-V/7-AAD) and $\Delta \Psi_{\mathrm{m}}\left(\mathrm{DiOC} \mathrm{C}_{6}\right)$ were obtained as described in Materials and methods. (B) Twenty-four hours later, total cell lysates were immunoblotted with antibodies against caspase-8, -9 and -3 or PARP. (C) Cells were harvested and the amounts of cytochrome $c$ and Bax in the cytosolic and mitochondrial fractions determined. The mitochondrial marker, COX-IV and the cytosolic marker, $\beta$-tubulin, were used to verify the purity of each fraction and were also used as loading controls. Cytosolic and mitochondrial fractionation was performed as described in Materials and methods. Results are representative of three independent experiments.

A

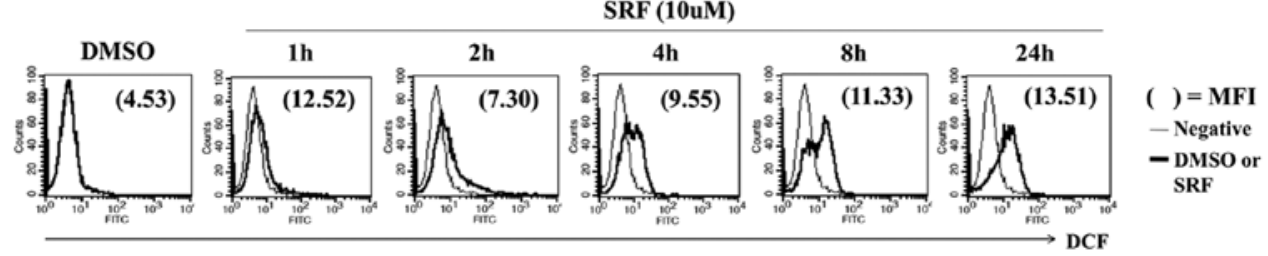

B
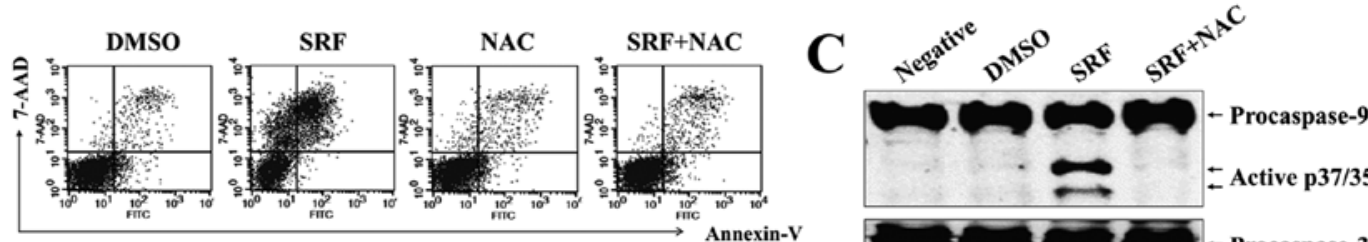

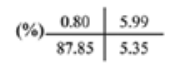

\begin{tabular}{l|c}
21.63 & 35.54 \\
\hline 42.03 & 0.80
\end{tabular}

\begin{tabular}{l|l}
2.22 & 8.31 \\
\hline 84.63 & 4.84
\end{tabular}

\begin{tabular}{c|c}
0.97 & 9.15 \\
\hline 83.19 & 6.68
\end{tabular}
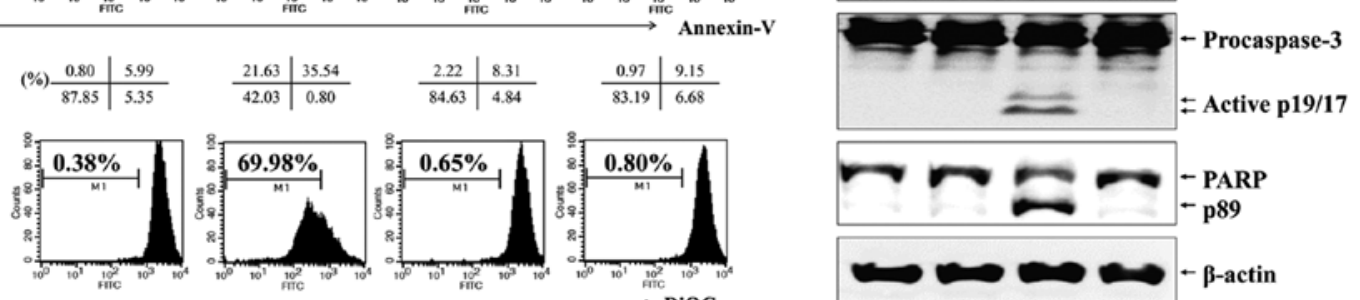

Figure 5. SRF induces ROS-dependent apoptosis in EBV-transformed B cells. (A) Cells were pretreated with $10 \mu \mathrm{M}$ DCFH-DA for 30 min and then treated with $10 \mu \mathrm{M}$ SRF for the indicated time periods. Cells were analyzed by flow cytometry. Numbers in the DCF histograms show the mean fluorescence intensity (MFI). Thin-line and thick-line histograms represent the ROS levels of negative control cells and SRF- or DMSO-treated cells, respectively. (B and C) Effects of NAC on SRF-induced apoptosis. To block ROS generation, cells were pretreated with NAC (10 mM) for $1 \mathrm{~h}$. Cells were then washed with PBS and treated with $10 \mu \mathrm{M}$ SRF or DMSO for $24 \mathrm{~h}$. (B) The number of apoptotic cells (Annexin-V/7-AAD) and $\Delta \Psi_{\mathrm{m}}\left(\mathrm{DiOC}_{6}\right)$ were obtained as described in Materials and methods. (C) Total protein was subjected to western blot analysis and immunoblotted against caspase-9, caspase-3, and PARP. Results are representative of three independent experiments. 
A

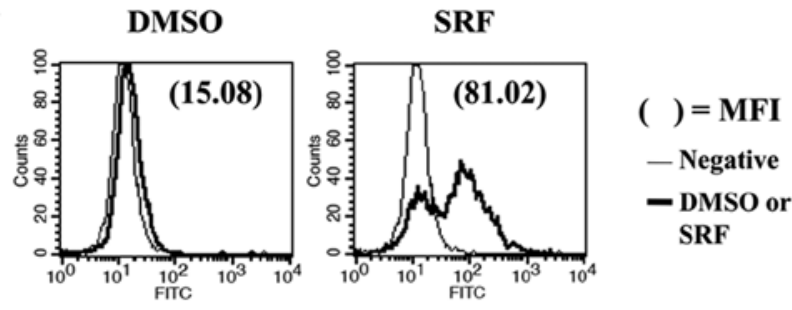

SRF

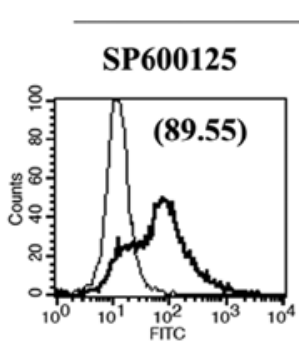

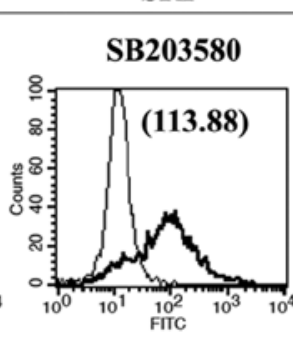

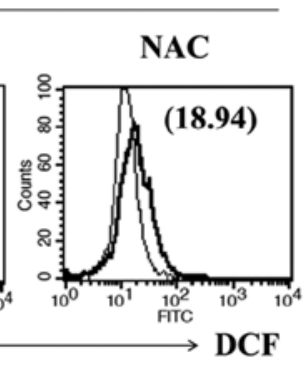

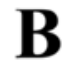
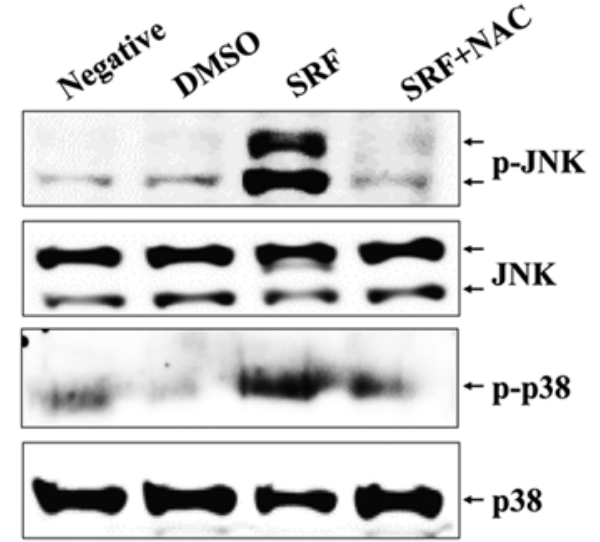

Figure 6. SRF induces JNK/p38-MAPK activation through ROS generation. Cells were pretreated with the inhibitors SP600125 (25 $\mu \mathrm{M})$, SB203580 (10 $\mu \mathrm{M})$ or NAC $(10 \mathrm{mM})$ for $1 \mathrm{~h}$. Cells were washed and treated with $10 \mu \mathrm{M}$ SRF or DMSO for $24 \mathrm{~h}$. (A) To measure ROS production, cells were pretreated with $10 \mu \mathrm{M}$ DCFH-DA for 30 min before treatment with SRF. Numbers in the DCF histograms indicate the mean fluorescence intensity (MFI). (B) Total protein was subjected to western blot analysis and successively immunoblotted for phospho-JNK and phospho-p38-MAPK. p38-MAPK and JNK were used as internal controls. Results are representative of three independent experiments.

(SRF, 35.54\%; with NAC, 9.15\%) and $\Delta \Psi_{\mathrm{m}}$ disruption (SRF, $69.98 \%$; with NAC, $0.80 \%)$. NAC also attenuated the activation of caspase- 9 and -3 , and the cleavage of PARP (Fig. 5C). SRF-induced ROS generation was blocked by NAC pretreatment, but not SP600125 or SB203580 treatment (Fig. 6A). NAC suppressed the SRF-induced phosphorylation of JNK/ p38-MAPK (Fig. 6B). These findings indicate that ROS plays an important role in SRF-induced activation of JNK/ p38-MAPK as well as apoptosis.

\section{Discussion}

It is well-established that extrinsic (death receptor) pathways and/or intrinsic (mitochondria) pathways contribute to chemotherapeutic drug-induced apoptosis in many cancer cells (27). One of the key events in the induction of mitochondrial pathway-mediated apoptosis is the disintegration of $\Delta \Psi_{\mathrm{m}}$, which induces the release of cytochrome $c$ and elicits caspase- 9 activation. This event is regulated by Bcl-2 family proteins (28). In particular, Bax translocation to mitochondria can change $\Delta \Psi_{\mathrm{m}}$. Bax plays an important role in inducing apoptosis in response to several stimuli (29). Bax is mostly cytosolic, but relocates to the mitochondria in response to stimuli (30). After translocation to the mitochondria, Bax, together with other pro-apoptotic Bcl-2 family members, including truncated $\mathrm{Bid}, \mathrm{Bad}$, and $\mathrm{Bak}$, induces the release of cytochrome $c$, AIF, endonuclease G, and Smac/DIABLO either by making pore channels by oligomerization in the outer mitochondrial membrane or by opening other channels $(28,31)$. Bax may be activated by phosphorylation of JNK and/ or p38-MAPK or by modifications in intracellular $\mathrm{pH}$. Due to its many tyrosine kinase targets, SRF has been reported to inhibit growth and induce apoptosis in preclinical models of human cancer (3). Here we found that SRF treatment resulted in the release of cytochrome $c$ from the mitochondria and induction of caspase- 9 and -3 activation in EBV-transformed $B$ cells. SRF decreased Bcl-2 expression and promoted Bax translocation to mitochondria (Fig. 2). These findings suggest that SRF treatment results in the generation of mitochondrial injury and caspase-dependent apoptosis in EBV-transformed $B$ cells.

Although originally identified as an inhibitor of the Raf/ MEK/Erk pathway (4), SRF is now known to function through diverse mechanisms in various tumor systems. We set out to scrutinize the mechanisms by which SRF induces apoptosis of EBV-transformed B cells. We demonstrated, for the first time, that SRF treatment induced apoptosis through activation of p38-MAPK in an ERK1/2-independent manner. Our results show that SRF treatment caused persistent activation of JNK and p38-MAPK rather than ERK1/2 in EBV-transformed B cells (Fig. 3A). Use of the JNK inhibitor SP600125 and p38-MAPK inhibitor SB203580 potently attenuated Bax translocation and afforded significant protection against SRF-induced apoptosis (Fig. 4). Proteins in the MAPK family contribute to various cellular responses. In particular, p38-MAPK and JNK play a pivotal role in the transmission of apoptotic signals $(32,33)$. Because the release of cytochrome $c$ from injured mitochondria represents a critical step in caspase activation, our finding that JNK and p38-MAPK activity was necessary for caspase activation implies that JNK and p38-MAPK may control some other mitochondrial-associated factor (e.g., Bax). In EBV-transformed B cells, SRF treatment caused downregulation of PI3K/Akt phosphorylation (Fig. 3B), similar to results obtained in human neuroblastoma cells and prostate cancer cells $(34,35)$. However, contrary to our expectations, there was no reduction in the phosphorylation level of ERK1/2. These results are consistent with those of recent studies that reported that SRF could induce apoptosis 
in melanoma and hepatocellular carcinoma cells through a MEK/ERK-independent mechanism $(36,37)$. Thus, it is clear that SRF has antitumor effects irrespective of its ability to inhibit p-ERK1/2.

Recent studies suggested that ROS may play a key role in apoptosis induction $(25,26)$. Oxidative stress can be elicited by sustained or aberrant ROS production and is associated with several biological events including apoptosis $(27,28)$. ROS is an important modulator of cellular signaling related to proliferation, apoptosis, and senescence (26). Several chemotherapeutic drugs exert their cytotoxic effects through the generation of ROS as a key mediator. Recent studies have reported that SRF might also be associated with ROS production $(29,30)$. Ample evidence indicates that chemically-mediated ROS production results in alteration of cellular functions and eventual apoptosis (26). Dysfunction of mitochondria induced by excessive ROS generation leads to dissipation of $\Delta \Psi_{\mathrm{m}}$ and apoptosis (26). ROS are also known to activate MAPKs (25). Our data indicated that sustained phosphorylation of JNK and p38-MAPK was caused by ROS generation after SRF treatment (Fig. 6). It is noteworthy that inhibition of ROS by NAC pretreatment ameliorated the effect of SRF on JNK and p38-MAPK phosphorylation, suggesting that SRF stimulates the production of ROS, which subsequently activate JNK and p38-MAPK, resulting in the translocation of Bax to mitochondria.

Taken together, we found that SRF inhibited cell growth and induced apoptosis in EBV-transformed B cells. SRF-induced apoptosis involved a reduction in $\mathrm{Bcl}-2$ expression and induction of Bax translocation to mitochondria, resulting in disruption of $\Delta \Psi_{\mathrm{m}}$ in EBV-transformed B cells. Our results also indicated that SRF elicited the activation of caspase-9 as the initiator caspase, followed by activation of caspase- 3 and -8. More importantly, ROS, JNK, p38-MAPK, and Bax participated in SRF-induced apoptosis. Consistent with this finding, ROS induced by SRF may act as upstream mediators of JNK and p38-MAPK signaling in EBV-transformed B cells treated with SRF (Fig. 5A). Furthermore, there is increasing evidence within the literature that ROS contribute to apoptosis caused by diverse stimuli. In conclusion, we demonstrated that SRF induces apoptosis of EBV-transformed B cells through ROS-dependent JNK/p38-MAPK signaling in an ERK-independent manner.

\section{Acknowledgements}

This study was supported by the 2014 Inje University Research Grant.

\section{References}

1. Young LS and Rickinson AB: Epstein-Barr virus: 40 years on. Nat Rev Cancer 4: 757-768, 2004.

2. Kuppers R: B cells under influence: transformation of B cells by Epstein-Barr virus. Nat Rev Immunol 3: 801-812, 2003.

3. Wilhelm S, Carter C, Lynch M, et al: Discovery and development of sorafenib: a multikinase inhibitor for treating cancer. Nat Rev Drug Discov 5: 835-844, 2006.

4. Wilhelm SM, Carter C, Tang L, et al: BAY 43-9006 exhibits broad spectrum oral antitumor activity and targets the RAF/ MEK/ERK pathway and receptor tyrosine kinases involved in tumor progression and angiogenesis. Cancer Res 64: 7099-7109, 2004.
5. Abou-Alfa GK, Schwartz L, Ricci S, et al: Phase II study of sorafenib in patients with advanced hepatocellular carcinoma. J Clin Oncol 24: 4293-4300, 2006.

6. Kane RC, Farrell AT, Saber H, et al: Sorafenib for the treatment of advanced renal cell carcinoma. Clin Cancer Res 12: 7271-7278, 2006.

7. Llovet JM and Bruix J: Molecular targeted therapies in hepatocellular carcinoma. Hepatology 48: 1312-1327, 2008.

8. Yang SY, Cui CL, Chi ZH, et al: Phase II clinical trial of sorafenib plus local chemotherapy in the treatment of metastatic renal cell carcinoma with pleural effusion. Zhonghua Yi Xue Za Zhi 92: 2998-3000, 2012 (In Chinese).

9. Galanis E, Anderson SK, Lafky JM, et al: Phase II study of bevacizumab in combination with sorafenib in recurrent glioblastoma (N0776): a north central cancer treatment group trial. Clin Cancer Res 19: 4816-4823, 2013.

10. Cervello M, Bachvarov D, Lampiasi N, et al: Novel combination of sorafenib and celecoxib provides synergistic anti-proliferative and pro-apoptotic effects in human liver cancer cells. PLoS One 8: e65569, 2013.

11. Wilhelm SM, Adnane L, Newell P, et al: Preclinical overview of sorafenib, a multikinase inhibitor that targets both Raf and VEGF and PDGF receptor tyrosine kinase signaling. Mol Cancer Ther 7: 3129-3140, 2008.

12. Huber S, Oelsner M, Decker T, et al: Sorafenib induces cell death in chronic lymphocytic leukemia by translational downregulation of Mcl-1. Leukemia 25: 838-847, 2011.

13. Lu X, Tang X, Guo W, et al: Sorafenib induces growth inhibition and apoptosis of human chondrosarcoma cells by blocking the RAF/ERK/MEK pathway. J Surg Oncol 102: 821-826, 2010.

14. Liu LP, Ho RL, Chen GG and Lai PB: Sorafenib inhibits hypoxia-inducible factor- $1 \alpha$ synthesis: implications for antiangiogenic activity in hepatocellular carcinoma. Clin Cancer Res 18: 5662-5671, 2012.

15. Reddy KB, Nabha SM and Atanaskova N: Role of MAP kinase in tumor progression and invasion. Cancer Metastasis Rev 22: 395-403, 2003.

16. Kim KN, Ham YM, Moon JY, et al: Acanthoic acid induces cell apoptosis through activation of the p38 MAPK pathway in HL-60 human promyelocytic leukaemia. Food Chem 135: 2112-2117, 2012.

17. Han X, Xu B, Beevers CS, et al: Curcumin inhibits protein phosphatases $2 \mathrm{~A}$ and 5 , leading to activation of mitogen-activated protein kinases and death in tumor cells. Carcinogenesis 33: 868-875, 2012.

18. Hyun MS, Hur JM, Mun YJ, et al: BBR induces apoptosis in HepG2 cell through an Akt-ASK1-ROS-p38MAPKs-linked cascade. J Cell Biochem 109: 329-338, 2010.

19. Rahmani M, Davis EM, Crabtree TR, et al: The kinase inhibitor sorafenib induces cell death through a process involving induction of endoplasmic reticulum stress. Mol Cell Biol 27: 5499-5513, 2007.

20. Reed JC: Bcl-2 family proteins: regulators of apoptosis and chemoresistance in hematologic malignancies. Semin Hematol 34: 9-19, 1997.

21. Adams JM and Cory S: The Bcl-2 protein family: arbiters of cell survival. Science 281: 1322-1326, 1998.

22. Chao DT and Korsmeyer SJ: BCL-2 family: regulators of cell death. Annu Rev Immunol 16: 395-419, 1998.

23. Wada T and Penninger JM: Mitogen-activated protein kinases in apoptosis regulation. Oncogene 23: 2838-2849, 2004.

24. Fresno Vara JA, Casado E, de Castro J, et al: PI3K/Akt signalling pathway and cancer. Cancer Treat Rev 30: 193-204, 2004.

25. Chiu WH, Luo SJ, Chen CL, et al: Vinca alkaloids cause aberrant ROS-mediated JNK activation, Mcl-1 downregulation, DNA damage, mitochondrial dysfunction, and apoptosis in lung adenocarcinoma cells. Biochem Pharmacol 83: 1159-1171, 2012.

26. Matsuzawa A and Ichijo H: Stress-responsive protein kinases in redox-regulated apoptosis signaling. Antioxid Redox Signal 7: 472-481, 2005.

27. Budihardjo I, Oliver H, Lutter M, et al: Biochemical pathways of caspase activation during apoptosis. Annu Rev Cell Dev Biol 15: 269-290, 1999.

28. Green DR: At the gates of death. Cancer Cell 9: 328-330, 2006.

29. Wei MC, Zong WX, Cheng EH, et al: Proapoptotic BAX and BAK: a requisite gateway to mitochondrial dysfunction and death. Science 292: 727-730, 2001.

30. Wolter KG, Hsu YT, Smith CL, et al: Movement of Bax from the cytosol to mitochondria during apoptosis. J Cell Biol 139: 1281-1292, 1997. 
31. Kuwana T, Mackey MR, Perkins G, et al: Bid, Bax, and lipids cooperate to form supramolecular openings in the outer mitochondrial membrane. Cell 111: 331-342, 2002.

32. Ghatan S, Larner S, Kinoshita Y, et al: P38 MAP kinase mediates Bax translocation in nitric oxide-induced apoptosis in neurons. J Cell Biol 150: 335-347, 2000

33. Tsuruta F, Sunayama J, Mori Y, et al: JNK promotes Bax translocation to mitochondria through phosphorylation of 14-3-3 proteins. EMBO J 23: 1889-1899, 2004.

34. Chai H, Luo AZ, Weerasinghe P, et al: Sorafenib downregulates ERK/Akt and STAT3 survival pathways and induces apoptosis in a human neuroblastoma cell line. Int J Clin Exp Pathol 3: $408-415,2010$
35. Oh SJ, Erb HH, Hobisch A, et al: Sorafenib decreases proliferation and induces apoptosis of prostate cancer cells by inhibition of the androgen receptor and Akt signaling pathways. Endocr Relat Cancer 19: 305-319, 2012.

36. Sánchez-Hernández I, Baquero P, Calleros L, et al: Dual inhibition of (V600E)BRAF and the PI3K/AKT/mTOR pathway cooperates to induce apoptosis in melanoma cells through a MEK-independent mechanism. Cancer Lett 314: 244-255, 2012.

37. Ou DL, Shen YC, Yu SL, et al: Induction of DNA damageinducible gene GADD45beta contributes to sorafenib-induced apoptosis in hepatocellular carcinoma cells. Cancer Res 70: 9309-9318, 2010 